\title{
Serum Levels of Brain-Derived Neurotrophic Factor in Patients with Obsessive-Compulsive Disorder in a Japanese Population
}

\author{
Takeyuki Akimoto1, Chihiro Sutoh1, Masaru Kuno², Daisuke Matsuzawa',2*, Tomihisa Niitsu', \\ Masaomi Iyo3 ${ }^{3}$, Eiji Shimizu, ${ }^{1,2}$
}

${ }^{1}$ Department of Cognitive Behavioral Physiology, Graduate School of Medicine, Chiba University, Chiba, Japan

${ }^{2}$ Research Center for Child Mental Development, Chiba University, Chiba, Japan

${ }^{3}$ Department of Psychiatry, Graduate School of Medicine, Chiba University, Chiba, Japan

Email: *dmatsuzawa@faculty.chiba-u.jp

How to cite this paper: Akimoto, T., Sutoh, C., Kuno, M., Matsuzawa, D., Niitsu, T., Iyo, M. and Shimizu, E. (2021) Serum Levels of Brain-Derived Neurotrophic Factor in Patients with Obsessive-Compulsive Disorder in a Japanese Population. Open Journal of Psychiatry, 11, 20-28. https://doi.org/10.4236/ojpsych.2021.111003

Received: December 7, 2020

Accepted: January 22, 2021

Published: January 25, 2021

Copyright (c) 2021 by author(s) and Scientific Research Publishing Inc. This work is licensed under the Creative Commons Attribution International License (CC BY 4.0).

http://creativecommons.org/licenses/by/4.0/

\begin{abstract}
A role of lower brain-derived neurotrophic factor (BDNF) content in the pathogenesis of several mental illnesses has been suggested, especially in major depression. It is not known whether BDNF is involved in the pathogenesis of obsessive-compulsive disorder (OCD). Herein, we assessed the serum BDNF content and its correlation with symptom severity in a Japanese population with OCD. The serum BDNF levels of OCD patients $(n=39)$ and healthy controls $(\mathrm{n}=37)$ were measured by ELISA. The severity of OCD symptoms was assessed by the Yale-Brown Obsessive-Compulsive Scale (Y-BOCS) and the Beck Depression Inventory (BDI). The OCD patients' BDNF levels were significantly higher than those of the controls $(17.5 \pm 7.3$ vs. $12.7 \pm 4.7)(\mathrm{p}<$ 0.01 ). No correlation was observed between the OCD patients' BDNF levels and their OCD symptoms as scored by the Y-BOCS. For all 76 subjects, the BDI scores were significantly negatively correlated with the serum BDNF levels. Our findings revealed that contrary to previous reports, the serum BDNF content in OCD patients could be higher than that of healthy subjects.
\end{abstract}

\section{Keywords}

Brain-Derived Neurotropic Factor (BDNF), Obsessive-Compulsive Disorder (OCD), ELISA, Depression

\section{Introduction}

Obsessive-compulsive disorder is a common psychiatric disease with the approximate lifetime prevalence of $1 \%-3 \%$ of adults [1]. Genetic epidemiological 
studies have demonstrated that OCD is a genetic multifactorial disorder, and there are biological risks that contribute to the onset of the disease with environmental stressors [2]. Brain-derived neurotrophic factor (BDNF) is a neurotrophin that promotes neuronal proliferation, viability and differentiation in the nervous systems. BDNF plays a particularly important role in the regulation of neural functions that respond to environmental stress events possibly through the hypothalamus-pituitary-adrenal (HPA) axis; BDNF also interacts with glucocorticoid [3]. Postmortem studies of human brains revealed that BDNF was involved in stress-related human psychopathologies such as depressive and anxiety disorders [4].

The serum BDNF level has been investigated as a biomarker of major depression in studies of the efficacy of antidepressant treatment [5] [6] and as a biomarker without diagnostic specificity [7]. The serum BDNF levels of patients with OCD have been reported in several studies. In two investigations that included un-medicated OCD patients, the serum BDNF levels were significantly lower in the OCD group compared to healthy controls [8] [9]. Wang et al. [10] assessed the plasma BDNF levels of OCD outpatients with 22 medication-free, 52 drug-treated, and 63 age- and gender-matched healthy controls in a Chinese population. In that study, the BDNF levels were lower in both medication-free and drug-treated OCD patients compared to normal controls. The same research group reported that plasma BDNF levels were lower in another group of OCD patients [11].

Interestingly, contrary to the studies of adult patients, a study of drug-naïve pediatric patients with OCD showed higher BDNF levels in the patients compared to healthy children [12], and another study with nearly drug-naïve OCD children did not observe this difference compared to healthy children [13]. We thus speculated that the serum level of BDNF in OCD patients may change over the course of the pathogenesis, and we then evaluated the serum BDNF protein levels in OCD patients. This study is also the first to assess the BDNF levels of Japanese OCD patients.

\section{Subjects and Methods}

\subsection{Subjects and Blood Samples}

Thirty-nine patients with OCD and 37 age-matched healthy individuals were enrolled in the study, with their written informed consent (Table 1). All of the OCD patients met with the OCD criteria of the Diagnostic and Statistical Manual of Mental Disorders, 4th Edition (DSM-IV) [14]. Patients who presented a comorbid Axis I diagnosis, a neurological disorder, a head injury, a serious medical condition or a history of drug or alcohol addiction were excluded. The study was approved by the Ethics Committee of Chiba University Graduate School of Medicine (Chiba, Japan) and performed in accordance with the Declaration of Helsinki II. All serum samples were collected between 9:00 a.m. and 3:00 p.m. and stored at $-80^{\circ} \mathrm{C}$ until use. 
Table 1. The clinical characteristics of the OCD patients and controls.

\begin{tabular}{cccc}
\hline & OCD patients & Healthy controls & p-value \\
\hline Age, yrs & $30.9 \pm 11.7(\mathrm{n}=39)$ & $29.9 \pm 9.1(\mathrm{n}=37)$ & $0.68^{\mathrm{a}}$ \\
Gender & & & $0.03^{\mathrm{b}}$ \\
Male & $14(36 \%)$ & $22(59 \%)$ & \\
Female & $25(64 \%)$ & $15(41 \%)$ & \\
Age at onset & $23.5 \pm 10.2(\mathrm{n}=36)$ & & $0.50^{\mathrm{c}}$ \\
Length of illness, yrs & $7.1 \pm 6.6(\mathrm{n}=36)$ & & $0.73^{\mathrm{c}}$ \\
Y-BOCS score & $22 \pm 7.6(\mathrm{n}=37)$ & & $0.10^{\mathrm{c}}$ \\
BDI score & $20 \pm 11.1(\mathrm{n}=33)$ & & $<0.01^{\mathrm{c}}$ \\
Use and dose of antidepressant(s) & $32(82 \%)$ & & $0.03^{\mathrm{c}}$ \\
BDNF (ng/ml) & $17.5 \pm 7.3$ & $12.7 \pm 4.7$ & $<0.01^{\mathrm{a}}$ \\
\hline
\end{tabular}

The data are mean $\pm \mathrm{SD}$, with $\mathrm{n}$-values and $\%$. By ${ }^{\mathrm{a}} \mathrm{t}$-test, ${ }^{\mathrm{b}} \mathrm{Chi}$-square test, or ${ }^{\mathrm{c}}$ test of no correlation with BDNF. BDI: Beck Depression Inventory. BDNF: brain-derived neurotrophic factor, OCD: obsessive-compulsive disorder, Y-BOCS: Yale-Brown Obsessive-Compulsive Scale.

\subsection{BDNF Measurements}

The serum BDNF levels of all of the subjects were measured using the BDNF Emax Immunoassay System kit (Promega, Madison, WI) according to the manufacturer's instructions. Briefly, 96-well plates were coated with anti-BDNF monoclonal antibody and incubated at $4^{\circ} \mathrm{C}$ for $18 \mathrm{hr}$. The plates were then incubated in a blocking buffer for $1 \mathrm{hr}$ at room temperature. The samples and BDNF standards were maintained at room temperature with shaking for $2 \mathrm{hr}$, followed by washing with the appropriate washing buffer. The plates were incubated with antihuman BDNF polyclonal antibody at room temperature for $2 \mathrm{hr}$, washed, and incubated with anti-IgY antibody conjugated to horseradish peroxidase for $1 \mathrm{hr}$ at room temperature. The plates were incubated in peroxidase substrate and tetramethylbenzidine solution to produce a color reaction. The reaction was stopped with $1 \mathrm{~mol} / \mathrm{L}$ hydrochloric acid, and the absorbance at 450 nm was measured with an Emax automated microplate reader (Molecular Devices, Tokyo).

\subsection{Statistical Analyses}

Student's t-test (two-tailed) was used for the comparison of age and BDNF levels between groups. The Chi-square test was used to verify the gender distributions within and between both groups. Tests of no correlation were used to identify the correlations among the age at onset, length of illness, Y-BOCS score, BDI score, and dose of antidepressant with the serum BDNF level. P-values $<0.05$ were accepted as significant. All of the statistical analyses were performed with SPSS software (ver. 17.0J, Tokyo).

\section{Results}

\subsection{The OCD Patients and Healthy Controls}

The characteristics of the OCD patients and healthy controls are summarized in 
Table 1. Regarding the 39 patients with OCD, age at onset and length of illness data were missing for three patients, Y-BOCS scores were missing for two patients, and the BDI scores were unavailable for six patients. The patients' and control subjects' ages were well-matched. In the OCD group, 14 patients were male (36\%) and 25 were female (64\%), whereas the healthy control group was comprised of $22(59 \%)$ males and $14(41 \%)$ females $(p=0.03)$.

\subsection{The BDNF Serum Level and Its Correlation with BDI Scores}

The BDNF serum levels were significantly higher in the OCD group (17.5 \pm 7.3$)$ compared to the control group $(12.7 \pm 4.7)(\mathrm{p}<0.01)$ (Figure 1$)$. There was no significant relationship between the BDNF serum levels and the ages of the subjects, the OCD patients' age at onset, the length of illness, the Y-BOCS score, or the use and dose of antidepressant(s). However, the BDI scores were significantly negatively associated with the BDNF serum levels $(\mathrm{p}<0.01)$ (Figure 2$)$ in OCD patients $(\mathrm{n}=33)$.

\section{Discussion}

To our knowledge, this is the first study to investigate the serum concentrations of BDNF in Japanese patients with OCD. Contrary to the most of the prior studies

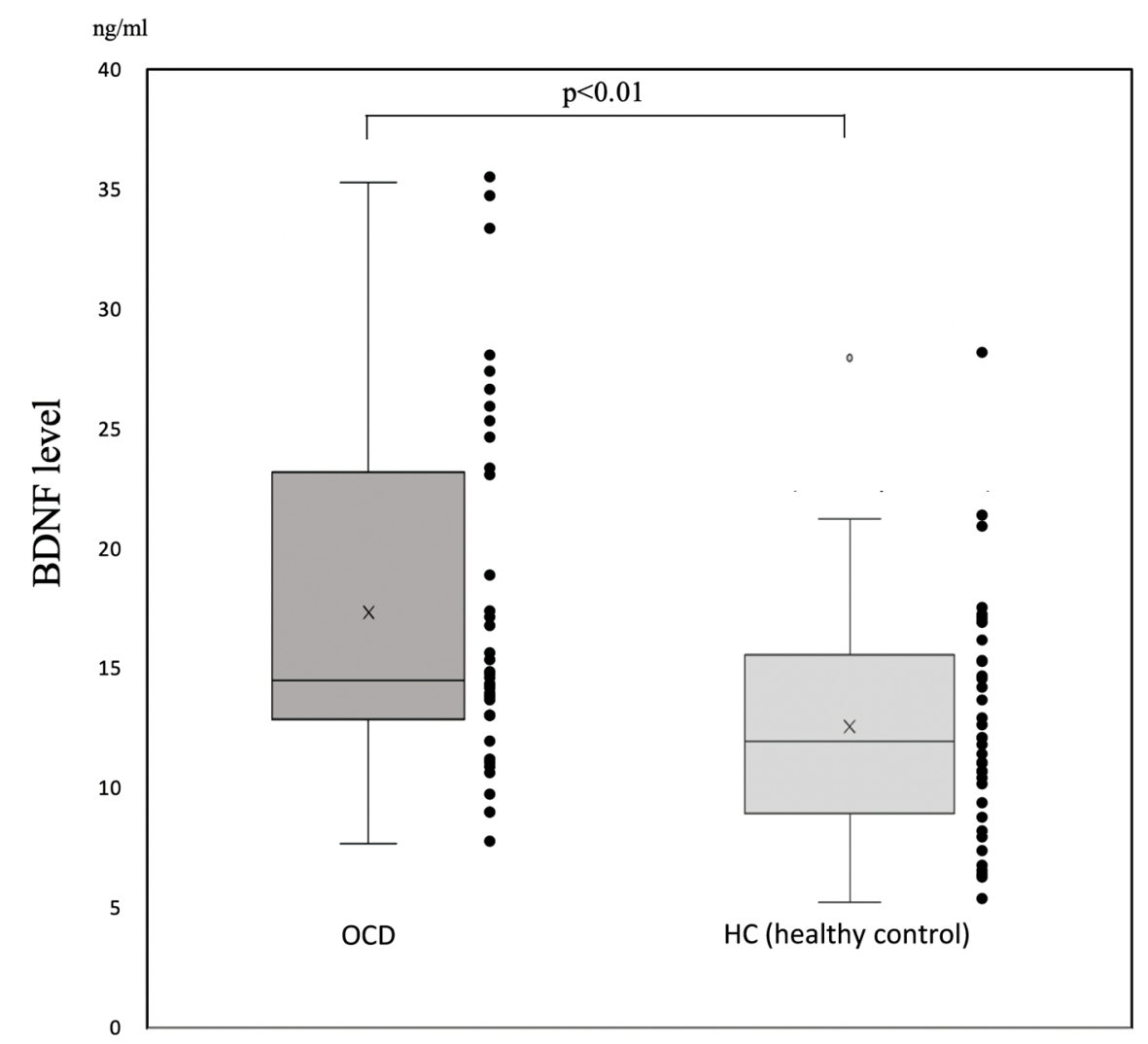

Figure 1. Box and scatter plot of serum BDNF levels. The serum BDNF levels were significantly higher in the OCD group (mean \pm SD $17.5 \pm 7.3$ ) compared to the healthy control (HC) group (mean \pm SD $12.7 \pm 4.7)(\mathrm{p}<0.01)$. 


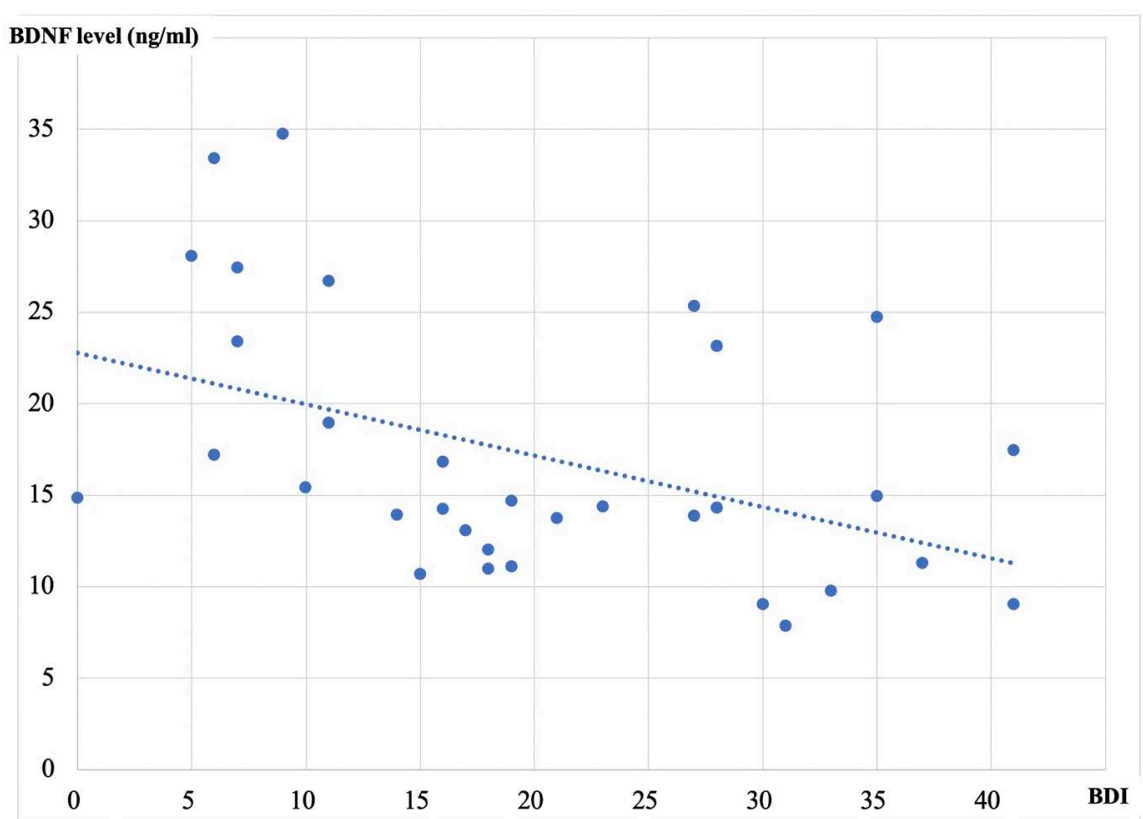

Figure 2. The correlation between the BDNF values and BDI scores for OCD patients. The BDI scores were significantly negatively associated with the BDNF serum levels ( $\mathrm{p}<$ $0.01)$ in OCD patients $(\mathrm{n}=33)$.

that measured BDNF in the serum of OCD subjects [8] [9] [10] [11], we observed the serum BDNF levels in the present OCD group were significantly higher than those in the control group. In only one previous study (examining children with OCD, mean age $12.4 \mathrm{yrs}$ ), the serum BDNF levels of the patients were higher than those of healthy controls [12].

A decreased level of BDNF compared to healthy levels has been reported to be very common in a range of mental diseases including major depression, posttraumatic stress disorder (PTSD) and anorexia nervosa, and BDNF has thus been considered an unspecific biomarker of neuropsychiatric illnesses [7]. Higher BDNF levels compared to controls were reported in bipolar disorder by several studies, and such higher levels were associated with longer illness duration [15], which indicated that the peripheral BDNF level would not necessarily be reduced in individuals with neuropsychiatric illnesses. Our present findings provide further evidence that the serum BDNF concentration could be high in specific states of illness such as OCD. As indicated by Simsek et al., OCD patients' increased BDNF levels might be adaptive in the early pathogenesis mediated by the HPA axis, with an increased secretion of glucocorticoids after acute stress [16].

Synaptic transmission via BDNF as a secretory molecule is involved in the mechanisms of neural plasticity, synaptogenesis, neurogenesis and cell survival in various regions of the human brain, and this transmission is regulated through the glutamate transmission pathways [17] [18]. In OCD, therefore, the concentration of peripheral BDNF could be high depending on the glutamatergic hyperactivity. Many studies have shown that the pathogenic profile of OCD is 
correlated with glutamatergic hyperactivity. For example, the level of glutamate in the cerebrospinal fluid of OCD patients was higher than that of healthy controls [19] [20]. Several agents that modulate glutamate via NMDA receptors (e.g., riluzole [21] [22], memantine [23] [24] [25], and ketamine [26]) have been shown to have potential efficacy for decreasing the severity of OCD symptoms.

In addition, magnetic resonance spectroscopy (MRS) studies of OCD patients have shown alterations of the glutamate concentration in the patients compared to healthy controls: specifically, a higher glutamate concentration in the orbitofrontal cortex (OFC) [27], a lower concentration in the caudate [28], and mixed results in the anterior cingulate cortex with both higher [29] and lower [30] concentrations being reported. These areas are involved in the cortico-striatalthalamo-cortical (CSTC) circuit dysfunction model of the pathogenesis of OCD. In line with the hypothesis, neuroimaging studies including functional magnetic resonance imaging (fMRI), PET, and SPECT examinations have revealed hyperactivity within the OFC, caudate, and thalamus [31].

It should be noted that most of our OCD patients were under medication (82\%, Table 1), whereas the patients enrolled in the previous studies were mostly drug-naïve or medication-free [3] [12]. In our study, the dosage of the antidepressant(s) used had no association with the serum level of BDNF (data not shown). The effects of medication taken by OCD patients should be taken into account, because a study of depressive patients indicated that the patients' serum BDNF levels could be altered with a good response to the drug treatment [32]. Two studies of the same group of OCD patients reported the patients' plasma BDNF levels, and in one of these studies, the patients were divided into drug-naïve and drug-treated groups; the plasma BDNF levels of both groups were significantly lower than those of healthy controls [10]. In the other study, the OCD patients' plasma BDNF levels were significantly lower than those of healthy controls, but the patients were not divided into subgroups based on their medication status, and no drug dosage information was provided in the report [11]. Whether or not the BDNF level studied in OCD patients is changed along with medication should be investigated in further studies.

Regarding the relationship between our OCD patients' BDNF levels and their symptoms, a significant negative relationship was revealed between the BDI scores and the BDNF levels in the OCD group (Figure 1), but the Y-BOCS results showed no relationship with the severity of OCD symptoms (data not shown). Thus, even though the present OCD group included patients with a depressive state, the OCD group's BDNF levels were higher than those of the healthy controls. In their OCD study, DosSantos et al. observed no correlation between the BDNF levels and the severity of depression [9]. The mean BDI score in their study (20.4) is almost the same as that obtained herein $(20 \pm 11.1, \mathrm{n}=$ 33). In another investigation, no significant relationships were observed between the BDNF levels and items on the Hamilton Rating Scale for Depression (17-items) (HAM-D-17) [8]. Because of the small sample size of each study (including ours), it remains difficult to make any conclusion regarding whether the 
BDNF level is altered in OCD patients in accord with their clinical manifestations.

In conclusion, this study is the first to examine the serum BDNF levels of Japanese patients with OCD, and the results of our analyses demonstrated that the serum BDNF levels of the OCD patients were significantly higher than those of healthy controls, and that the serum BDNF levels were significantly correlated with the patients' depressive state.

\section{Conflicts of Interest}

The authors declare no conflicts of interest regarding the publication of this paper.

\section{References}

[1] Kessler, R.C., et al. (2005) Lifetime Prevalence and Age-of-Onset Distributions of DSM-IV Disorders in the National Comorbidity Survey Replication. Archives of General Psychiatry, 62,593-602. https://doi.org/10.1001/archpsyc.62.6.593

[2] Grisham, J.R., Anderson, T.M. and Sachdev, P.S. (2008) Genetic and Environmental Influences on Obsessive-Compulsive Disorder. European Archives of Psychiatry and Clinical Neuroscience, 258, 107-116. https://doi.org/10.1007/s00406-007-0789-0

[3] Suliman, S., Hemmings, S.M. and Seedat, S. (2013) Brain-Derived Neurotrophic Factor (BDNF) Protein Levels in Anxiety Disorders: Systematic Review and Meta-Regression Analysis. Frontiers in Integrative Neuroscience, 7, 55. https://doi.org/10.3389/fnint.2013.00055

[4] Duman, R.S. and Monteggia, L.M. (2006) A Neurotrophic Model for Stress-Related Mood Disorders. Biological Psychiatry, 59, 1116-1127. https://doi.org/10.1016/j.biopsych.2006.02.013

[5] Kishi, T., et al. (2017) Brain-Derived Neurotrophic Factor and Major Depressive Disorder: Evidence from Meta-Analyses. Frontiers in Psychiatry, 8, 308. https://doi.org/10.3389/fpsyt.2017.00308

[6] Bocchio-Chiavetto, L., et al. (2010) Serum and Plasma BDNF Levels in Major Depression: A Replication Study and Meta-Analyses. The World Journal of Biological Psychiatry, 11, 763-773. https://doi.org/10.3109/15622971003611319

[7] Bandelow, B., et al. (2017) Biological Markers for Anxiety Disorders, OCD and PTSD: A Consensus Statement. Part II: Neurochemistry, Neurophysiology and Neurocognition. The World Journal of Biological Psychiatry, 18, 162-214. https://doi.org/10.1080/15622975.2016.1190867

[8] Maina, G., et al. (2010) Serum Levels of Brain-Derived Neurotrophic Factor in Drug-Naive Obsessive-Compulsive Patients: A Case-Control Study. Journal of Affective Disorders, 122, 174-178. https://doi.org/10.1016/j.jad.2009.07.009

[9] Dos Santos, I.M., et al. (2011) Symptom Dimensional Approach and BDNF in Unmedicated Obsessive-Compulsive Patients: An Exploratory Study. CNS Spectrums, 16, 179-189. https://doi.org/10.1017/S1092852912000363

[10] Wang, Y., et al. (2011) Brain-Derived Neurotrophic Factor (BDNF) Plasma Levels in Drug-Naive OCD Patients are Lower than Those in Healthy People, But are Not Lower than Those in Drug-Treated OCD Patients. Journal of Affective Disorders, 133, 305-310. https://doi.org/10.1016/j.jad.2011.04.002 
[11] Wang, Y., et al. (2015) BDNF Val66Met Polymorphism and Plasma Levels in Chinese Han Population with Obsessive-Compulsive Disorder and Generalized Anxiety Disorder. Journal of Affective Disorders, 186, 7-12. https://doi.org/10.1016/j.jad.2015.07.023

[12] Simsek, S., et al. (2016) Cortisol and Brain-Derived Neurotrophic Factor Levels Prior to Treatment in Children With Obsessive-Compulsive Disorder. Journal of Clinical Psychiatry, 77, e855-e859. https://doi.org/10.4088/JCP.15m10146

[13] Colak Sivri, R., Bilgic, A. and Kilinc, I. (2018) Cytokine, Chemokine and BDNF Levels in Medication-Free Pediatric Patients with Obsessive-Compulsive Disorder. European Child \& Adolescent Psychiatry, 27, 977-984. https://doi.org/10.1007/s00787-017-1099-3

[14] Association, A.P. (1994) Diagnostic and Statistical Manual of Mental Disorders. 4th Edition (DSM-IV). American Psychiatric Press, Washington, DC.

[15] Munkholm, K., Vinberg, M. and Kessing, L.V. (2016) Peripheral Blood Brain-Derived Neurotrophic Factor in Bipolar Disorder: A Comprehensive Systematic Review and Meta-Analysis. Molecular Psychiatry, 21, 216-228.

https://doi.org/10.1038/mp.2015.54

[16] Tapia-Arancibia, L., et al. (2004) Physiology of BDNF: Focus on Hypothalamic Function. Frontiers in Neuroendocrinology, 25, 77-107. https://doi.org/10.1016/j.yfrne.2004.04.001

[17] Vasquez, C.E., et al. (2014) NMDA Receptor Dysregulation in Chronic State: A Possible Mechanism Underlying Depression with BDNF Downregulation. Neurochemistry International, 79, 88-97. https://doi.org/10.1016/j.neuint.2014.09.007

[18] Panja, D. and Bramham, C.R. (2014) BDNF Mechanisms in Late LTP Formation: A Synthesis and Breakdown. Neuropharmacology, 76, 664-676. https://doi.org/10.1016/j.neuropharm.2013.06.024

[19] Bhattacharyya, S., et al. (2009) Anti-Brain Autoantibodies and Altered Excitatory Neurotransmitters in Obsessive-Compulsive Disorder. Neuropsychopharmacology, 34, 2489-2496. https://doi.org/10.1038/npp.2009.77

[20] Chakrabarty, K., et al. (2005) Glutamatergic Dysfunction in OCD. Neuropsychopharmacology, 30, 1735-1740. https://doi.org/10.1038/sj.npp.1300733

[21] Grant, P., et al. (2007) An Open-Label Trial of Riluzole, a Glutamate Antagonist, in Children with Treatment-Resistant Obsessive-Compulsive Disorder. Journal of Child and Adolescent Psychopharmacology, 17, 761-767. https://doi.org/10.1089/cap.2007.0021

[22] Coric, V., et al. (2005) Riluzole Augmentation in Treatment-Resistant ObsessiveCompulsive Disorder: An Open-Label Trial. Biological Psychiatry, 58, 424-428. https://doi.org/10.1016/j.biopsych.2005.04.043

[23] Haghighi, M., et al. (2013) In a Double-Blind, Randomized and Placebo-Controlled Trial, Adjuvant Memantine Improved Symptoms in Inpatients Suffering from Refractory Obsessive-Compulsive Disorders (OCD). Psychopharmacology, 228, 633-640. https://doi.org/10.1007/s00213-013-3067-z

[24] Ghaleiha, A., et al. (2013) Memantine Add-on in Moderate to Severe Obsessive-Compulsive Disorder: Randomized Double-Blind Placebo-Controlled Study. Journal of Psychiatric Research, 47,175-180. https://doi.org/10.1016/j.jpsychires.2012.09.015

[25] Stewart, S.E., et al. (2010) A Single-Blinded Case-Control Study of Memantine in Severe Obsessive-Compulsive Disorder. Journal of Clinical Psychopharmacology, 30, 34-39. https://doi.org/10.1097/JCP.0b013e3181c856de 
[26] Rodriguez, C.I., et al. (2013) Randomized Controlled Crossover Trial of Ketamine in Obsessive-Compulsive Disorder: Proof-of-Concept. Neuropsychopharmacology, 38, 2475-2483. https://doi.org/10.1038/npp.2013.150

[27] Whiteside, S.P., et al. (2006) A Magnetic Resonance Spectroscopy Investigation of Obsessive-Compulsive Disorder and Anxiety. Psychiatric Research, 146, 137-147. https://doi.org/10.1016/j.pscychresns.2005.12.006

[28] Rosenberg, D.R., et al. (2000) Decrease in Caudate Glutamatergic Concentrations in Pediatric Obsessive-Compulsive Disorder Patients Taking Paroxetine. Journal of the American Academy of Child \& Adolescent Psychiatry, 39, 1096-1103. https://doi.org/10.1097/00004583-200009000-00008

[29] Yucel, M., et al. (2008) Anterior Cingulate Glutamate-Glutamine Levels Predict Symptom Severity in Women with Obsessive-Compulsive Disorder. Australian \& New Zealand Journal of Psychiatry, 42, 467-477. https://doi.org/10.1080/00048670802050546

[30] Rosenberg, D.R., et al. (2004) Reduced Anterior Cingulate Glutamatergic Concentrations in Childhood OCD and Major Depression versus Healthy Controls. Journal of the American Academy of Child \& Adolescent Psychiatry, 43, 1146-1153. https://doi.org/10.1097/01.chi.0000132812.44664.2d

[31] Frydman, I., et al. (2016) Can Neuroimaging Provide Reliable Biomarkers for Obsessive-Compulsive Disorder? A Narrative Review. Current Psychiatry Reports, 18, 90. https://doi.org/10.1007/s11920-016-0729-7

[32] Shimizu, E., et al. (2003) Alterations of Serum Levels of Brain-Derived Neurotrophic Factor (BDNF) in Depressed Patients with or Without Antidepressants. Biological Psychiatry, 54, 70-75. https://doi.org/10.1016/S0006-3223(03)00181-1 